\title{
PRESCRIPTION PATTERN OF ANTIMALARIAL DRUGS IN CHILDREN BELOW 5 YEARS IN A TERTIARY HEALTH INSTITUTION IN NIGERIA
}

\author{
E. U. Etuk ${ }^{1}$, M. A. Egua ${ }^{2}$ and A. A. Muhammad ${ }^{3}$ \\ ${ }^{1}$ Department of Pharmacology, College of Health Sciences, Usmanu Danfodiyo University, Sokoto, Nigeria \\ ${ }^{2}$ Department of Medicine, Specialist Hospital Sokoto,, Nigeria \\ ${ }^{3}$ Pharmacy Department, Specialist Hospital Sokoto, Nigeria \\ Reprint requests to: Dr. E. Etuk, C/o P.O. Box 2298, Sokoto, Nigeria. E-mail: etuk2005@yahoo.co.uk
}

\begin{abstract}
Background: This study examines the diagnosis of malaria and pattern of prescription of antimalarial drugs in the most vulnerable age group (the under 5 children) in the study environment in order to identify the possible shortcomings and suggest solutions so as to improve the treatment outcome in future.

Methods: The hospital records of 430 children with malaria infection admitted for treatment in a chosen tertiary health facility between January to December 2005 were selected for study. Forty-eight case records were excluded due to incomplete information. Data on demographic, clinical features of disease, diagnostic procedures, drug administration and the treatment out come were collected from the patients' records.

Results: Analysis of the data revealed that more male (213) than female (169) children were admitted for malaria treatment: Fever with convulsion (55.8\%) was the commonest presenting symptoms, and anemia was the most frequent complications of malaria recorded. Chloroquine was found to be the most prescribed antimalarial agent and overall antemisinin-based drug was prescribed either as a first or second line treatment in only $18.2 \%$ of the cases. The death rate recorded was $16 \%$.

Conclusion: The pattern of antimalarial drugs prescription in the study center in most cases did not meet the recommended guidelines. The prescriptions were predominantly chloroquine, instead of artemisinin based. The death rate was comparatively high. Measures to raise the level of awareness among the practitioners on the current National policy on malaria treatment through seminars and workshops were suggested.
\end{abstract}

Key words: Malaria, artemisinin, convulsion, children

\footnotetext{
Résumé

Introduction: Cette étude examine le diagnostic du paludisme et le modèle de l'ordonnance des médicaments antipaludéens pour le groupe d'âge le plus vulnérable (les enfants de moins de cinq ans), dans le but d' identifier les défaillances possibles et alors suggérer des solutions pour ainsi améliorer les soins dans l'avenir.

Méthodes: L'étude a été basée sur les données de l'hôpital portant sur 430 enfants atteints du paludisme et hospitalisés dans des centres médicaux sélectionnés de troisième catégorie, entre Janvier et Décembre, 2005. Quarante huit cas ont été exclus pour l'insuffisance d'information. Les données démographiques, les symptômes des maladies, les procédures du diagnostic, l'administration de médicaments et le résultat des traitements ont été collectés du registre des malades.
} 
Résultats: L'analyse des données a révélé que plus des garçons (213) que des filles (169) ont été hospitalisés pour les traitements du paludisme. La fièvre avec convulsions (55,8 pour cent) était plus fréquente tandis que l'anémie était la complication paludéenne la plus notée. II a été aussi noté que la chloroquine a été le médicament antipaludéen le plus prescrit et que les médicaments spécialisés à base de l'Artémisinine ont été, en tout et pour tout, prescrits en première et en deuxième positions de traitement couvrant ainsi 18,2 pour cent seulement des cas. Le taux de mortalité était à 16 pour cent.

Conclusion: Le modèle de la prescription des médicaments antipaludéens, dans le centre de cette étude, n'a pas atteint le niveau des recommandations dans beaucoup de cas. Les prescriptions étaient en majorité la chloroquine au lieu des médicaments spécialisés a base Artémisinine. Le taux de mortalité est relativement élevé. Des mesures d'intensifier la sensibilisation pour les praticiens a travers séminaire et ateliers sur la Nouvelle Politique Nationale sur le traitement du paludisme ont été suggérées.

Mots clés: Paludisme, artémisinine, agitation, enfants

\section{Introduction}

The reduction of deaths associated with malaria in children especially in the sub-Saharan Africa is a realistic and attainable goal if the management of the infection is improved. The malarial control programmes both in Africa and elsewhere consist of treatment of infected persons with animalarial drugs and the prevention of transmission of the disease by avoiding bites from the carrier mosquitoes. The proper control of malaria infection globally is being threatened on an unprecedented scale by rapidly growing resistance of Plasmodium falciparum to conventional monotherapies such as chloroquine, sulphadoxine - pyrimethamine and amodiaquine. ${ }^{1}$ As a response to the anti-malarial drug resistance situation, the World Health Organisation (WHO) recommends that treatment policies for falciparum malaria in all countries experiencing resistance to monotherapies should be combination therapies, preferably those containing an arteminisin derivative (ACT-artemisinin-based combination therapy). ${ }^{1}$

Over the last five years, many countries in the world including Nigeria have modified their treatment policies to include ACTs as 1 st - line or 2nd line drugs for the treatment of malaria. But haphazard implementation of this policy by health care providers may jeopardize the achievement of its laudable goal. Equally relevant in the success of this malaria war, is the WHO position on the diagnosis and treatment of malaria in rural areas that has been grossly misapplied. The WHO advises that; in areas where malaria is endemic, recognition of fever and its presumptive treatment with antimalarial drugs is essential part of the strategy to reduce the morbidity and mortality due to this disease. ${ }^{2}$ This policy did not undermine the importance of conducting laboratory investigations to confirm the clinical diagnosis of malaria.

The focus of the current study is to examine the diagnosis of malaria and pattern of prescription of anti-malarial drugs in the vulnerable age group (the under fives) in a tertiary health setting for the period January to December, 2005. The level of compliance with recommended therapy guidelines will be assessed and possibly correlation drawn between the cases management and treatment outcome.

\section{Materials and Methods}

This was cross-sectional retrospective study conducted in the Department of Paediatrics, Specialist Hospital, Sokoto, northwestern Nigeria. The hospital records of children under the age of 5 years admitted during the period, January to December 2005 were collected and examined. The records of all the children within the considered age- group diagnosed, admitted and managed, as malarial cases were isolated, screened, and relevant data extracted using a pre-piloted data collection form.

The diagnosis of malaria in the cases selected were based on the WHO's clinical case definition for malaria: fever or history of fever without obvious cause supported with or without thin/thick blood film microscopic examination for malaria parasites. Among the information considered during data collection were: age, sex and weight of the patients; symptoms presented at the time of admission; complications associated with the disease; and the method of confirmation of the diagnosis.

The type(s) of antimalarial drugs prescribed during the course of admission, dosage administered and the duration of administration, response to treatment (as measured by the disappearance of symptoms and duration of stay in the hospital) and records of laboratory investigations carried out were also charted. The final outcome (survived, death) was recorded. Excluded from this study were records of children with other established illnesses like measles, pneumonia etc. being treated with antimalarial drugs. The data collected were analyzed using statistical program for social sciences (SPSS) software. 


\section{Results}

The hospital records of 430 children of ages 0 to 5 years admitted for treatment of malaria during the study period were examined and 382 selected. Forty eight $(11.2 \%)$ case records were excluded from the study due to incomplete information. Analysis of the data collected indicates that; more male (213) than female (169) children were admitted for malaria treatment. This gives a male to female ratio of 1.26:1. The age distribution of the affected children were: 0 1 year $=113(29.6 \%) ;>1-2$ years $=202$ (52.9\%) and $>2-5$ years $=67(17.5 \%)$ giving the most affected age group as greater than one to two years (Table 1). Of significant interest were two infants $(0.5 \%)$, age less than 3 months diagnosed, admitted and treated for malaria. The thick/thin blood smear film microscopy for malarial parasites was carried out in only $26(6.8 \%)$ of the 382 children diagnosed clinically to have malaria. Fever with convulsion (226; $55.8 \%)$ was the commonest presenting symptoms, and anemia (20.7\%) was the most common complication of malaria. Sixty deaths (16\%) were recorded, giving a death rate of about 1 per every 6 children admitted.

Table 1. Summary of the clinical data of 430 patients

\begin{tabular}{ll}
\hline Description & No. (\%) \\
\hline Total Number of cases & 430 \\
Number of selected cases & $382(88.8)$ \\
Number of cases excluded & $48(11.2)$ \\
Age group (years) & \\
$0-1$ & $113(29.6)$ \\
$>1-2$ & $203(52.9)$ \\
$>2-5$ & $67(17.5)$ \\
Presenting features & \\
Fever alone & $92(24.2)$ \\
Fever, vomiting and diarrhea & $63(16.4)$ \\
Fever and convulsion & $213(55.8)$ \\
Diarrhea and vomiting & $14(3.8)$ \\
Sex & \\
M & $213(55.8)$ \\
F & $169(42.2)$ \\
M:F ratio & $1.26: 1$ \\
Complications & $79(20.7)$ \\
Anaemia & $15(3.9)$ \\
Cerebral Malaria & $27(7.1)$ \\
Respiratory distress & $43(11.3)$ \\
Dehydration & $60(16)$ \\
Death & \\
\hline
\end{tabular}

The records of antimalarial agent prescribed as a 1st line monotherapy were: chloroquine (CQ) 7.1\%; sulphadoxine/pyrimethamine (SP) $1.3 \%$; quinine hydrochloride (14.6\%); dihydroartemisinin (DHA)
(0.8\%); artemether/lumefantrine $(\mathrm{AL}) \quad(1.8 \%)$; artesunate/amodiaquine (AA) (2.1\%) and artemether (5.0\%). The following antimalarial agents were prescribed as a second line therapy following the failure of choroloquine. Sulphadoxine/pyrimethamine $\quad(10.2 \%)$; quinine (23.8\%); dihydroartemisinin (DHA) (5.2\%); artemether/lumefantrine (AL) (14.3\%) and artemether (13.9\%). The artemisinin based combination therapy (ACT) was only prescribed in $18.2 \%$ of the cases, either as a first or second line drugs. The ACT prescribed were those, such as $A L$ and $A A$, formulated and marketed as a fixed combined dose therapy. Overall, inadequate dosages of chloroquine (considering the quantity, frequency, duration) were prescribed in $83.8 \%$ of the cases.

The weights of the children were not recorded. The duration of stay by the patients in the hospital were $1-3$ days (216; 56.6\%), 3-6 days (120; 31.3\%) and 7 days and above (46; 12.1\%) which gives 4.5 days as the mean duration of stay in hospital.

Table 2. Prescription pattern of antimalarial drugs in 430 patients

\begin{tabular}{ll}
\hline Drugs & No. (\%) \\
\hline Chloroquine & $27(7.1)$ \\
SP & $5(1.3)$ \\
Quinine & $56(14.6)$ \\
DHA & $3(0.8)$ \\
AL & $7(1.8)$ \\
AA & $8(2.1)$ \\
Artemeter & $19(5)$ \\
CQ; SP & $39(10.2)$ \\
CQ; Quinine & $91(23.8)$ \\
CQ; DHA & $20(5.2)$ \\
CQ; AL & $55(14.3)$ \\
CQ; Artemeter & $53(13.9)$ \\
\hline
\end{tabular}

SP: Sulphadoxine + pyrimethamine; DHA: Dihydroxyartemisinin; AL: Artemether + lumefantrine; AA: Artesunate + amodiaquine; $\mathrm{CQ}$ : Chloroquine

\section{Discussion}

The 382 patients selected for this study represents only a fraction of the cases of malaria infection seen in the center during the study period. The majority of children who presented with what was considered as mild degree of malaria infection were always treated and discharged as out- patients. There was a significant number of missing and incomplete records. Despite all these, the number of malaria cases, age 0-5 years admitted within one year in this hospital was comparatively higher than the 583 children, aged 0 to 10 years recorded in a similar study at the center de Hospitalier de Libreville, Gabon over a two year period. ${ }^{3}$ This may be as a result of a higher transmission rate of malaria 
parasite in the environment or the differences in population density.

Table 1 indicates a higher incidence of malaria infection among the male children. Malaria is not known to be associated with any sex preference but several studies have reported a higher incidence in male. $^{3,4}$ The higher incidence among the male children may be a mere reflection of the sex distribution in the study population or an indication of a stronger immunity developed by the female children. The most affected age group was $>1-2$ years. This seems to agree with the previous reports: Severe malaria attacks develop in children between the ages of 9 months and 2 years, thereafter increasing active immunity develops so that malaria attack becomes less frequent. ${ }^{5,6}$ Before now, it was rare to have severe malaria in children less than 6 months born to immune mothers and being fed exclusively on breast milk. This study has shown that two children $(0.5 \%)$ age less than 3 months were admitted for treatment of malaria. This may be as a result of the increase transmission rate of malaria parasites in this environment. . Several studies have reported progressive increase in mean parasite clearance time, elevated mean parasite density and decrease sensitivity to antimalaria therapy in this environment. $4,7,8$

The thick/thin blood film microscopy examination for malaria parasites was conducted in only $6.8 \%$ of the 382 selected patients. The study center is a tertiary health facility where a more specific diagnosis is required. Studies have shown that physician diagnosed correctly $86 \%$ of cases of malaria and $61 \%$ of cases of other febrile illnesses on clinical grounds without the results of blood film examination. ${ }^{9}$ Also when fever or history of fever is used as the sole criteria for diagnosis of malaria, over diagnosis is frequently substantial, especially during periods of low malaria transmission. ${ }^{10}$ When nearly all malaria parasites were sensitive to chloroquine, a cheap and safe drug, this was of little importance and it was reasonable to treat with this drug all patients with any suspicion of malaria. However, this is no longer the case and it is becoming increasingly necessary, even in tropical Africa, to choose a more expensive drug for the first line treatment of malaria. In such circumstances, accurate diagnosis becomes increasingly important. ${ }^{9}$ The introduction of malaria antigen detection in blood using commercially available assays has further lessened the burden of confirming malaria diagnosis through blood film microscopy even though the later is still considered as the gold standard.

The common presentation of malaria found in this study was fever with convulsion $(55.8 \%$ ) (Table 1). The Presentations of severe malaria are different at different ages and in areas with different levels of transmission. ${ }^{3}$ Fever is a characteristic feature of $P$. falciparum infection, but about $3.8 \%$ of the patients recorded in this study were afebrile on admission presenting instead with vomiting and diarrhea. Home medication with antipyretic or antimalarial agents may be responsible for the masking of the fever and such cases may be misdiagnosed when clinical parameters alone are used. Also the mortality rate is indicated as $16 \%$ ( 60 deaths/382 cases). This is high when compared to $8.9 \%$ (52 deaths/582 cases) reported by Arnaud, et al 2005 among the Gabonese children, but in line with studies from other geographic areas where mortality rates range between 8 and $40 \% .{ }^{11,12}$

The finding that, children were not constantly weighed before administration of antimalarial drugs was equally important. Victims of frequent malaria attacks are often deprived children with low weight and stunted growth. Thus, the determination of their actual weight is necessary to avoid administration of subtherapeutic dose of the drug which may result in therapeutic failure and/or development of resistance to the drug while over dosage can cause toxicity.

Chloroquine was found to be the most prescribed antimalarial agent despite several reports of increasing level of resistance to chloroquine by the parasites in this environment. ${ }^{4,8}$ This may cause a high number of therapeutic failures. Artemisinin based antimalarial agent was prescribed either as a first or second line drug only in $18.2 \%$ of the cases. This might not be unconnected with the high cost of the artemisinin compared to chloroquine. The use of combination therapy has been reported to be severely restricted by cost especially in sub-Saharan Africa. ${ }^{13}$ In $68.4 \%$ of the antimalarial prescriptions examined, a second antimalarial drug was only introduced sequel to the failure of the first agent. There was a replacement rather than combination therapy as emphasized by relevant guidelines. The combined therapy used was only those formulated and marketed as fixed combined dose therapy.

Assessment of the prescription pattern of antimalarial drugs in the study center based on the current World Health Organization and the Nigerian national policies on malaria has left a very unsatisfactory outcome. The WHO Technical consultation committee strongly endorsed the potential of combination therapy for use in Africa. It recommended the following four combination therapies with potential available safety and efficacy data, which are listed below in prioritized order:

1. Artemether/lumefantrine

2. Artesunate/amodiaquine

3. Artesunate (AS) plus sulphadoxine/pyrimethamine (SP)

4. Amodiaquine plus sulphadoxine/pyrimethamine

The options not recommended for policy include:

1. Chloroquine based combinations $(C Q+S P$ and $C Q+A S)$ 
2. One day treatment of artesunate $+\mathrm{SP}$

3. Mefloquine based combinations (mefloquine + artesunate)

The pattern of antimalarial prescriptions as seen in the study center is dominated by chloroquinebased combinations.

In conclusion, this study has identified fever and convulsion as the commonest presenting symptoms and anemia as the most frequent complication of malaria in under- five age children admitted for treatment in the center during the study period. The death rate from the disease was high (16\%) and the prescription of artemisinin based antimalarial drugs was low (18.2\%). There is therefore an urgent need to raise the awareness level of the health service providers concerning the current National policy on malaria treatment. This can be achieved through regular organization of seminars and workshops to refresh the medical workers on current approaches concerning the management of malaria infection in under five years children. Additional measure like the provision of free antimalarial drugs for children of this age group may improve the treatment outcome and further reduce the death rate from the disease.

\section{Acknowledgments}

The Authors are grateful to $\mathrm{Dr}$ N. M. Jiya of Paediatrics Department, UDUTH, and the staff of The Records, and Paediatrics Departments, Specialist Hospital, Sokoto, Nigeria for their contributions to this paper.

\section{References}

1. World Health Organisation. The use of Antimalarial drugs. Position of WHO's roll back malaria department on malaria treatment policy, WHO unpublished report. 2003. WHO/CDS/RBM/2003. 26.

2. World Health Organisation. Antimalaria drug combination therapy. Report of a WHO's technical consultation committee. WHO unpublished 2001. WHO/CDS/RBM/2001.35.

3. Arnaud D-E, Pascal C, Obiang N, et al. Severe falciparum malaria in Gabonese Children: Clinical and Laboratory features. Malar J. 2005;4:14751486.

4. Bello SO. Moh'd BY, Uktu AL, Amadu BM, Adeniyi $A A$, Chuma JY. The pattern of infection and in vivo response to chloroquine by uncomplicated plasmodium falciparun malaria in notherwestern Nigeria. Afr J Biotechnol. 2004;4:79-82.

5. Diempsey TMO. Malaria in children. Africa Health (Suppl.) 2001; $21-23$.

6. Kenan PG, Krisna S. Antimalaria combinations. Lancet. 2004;364:285-294.

7. Osisanya JO. The role of chemotherapy in the control of malaria in Africa. Nigerian Medical Practitioner. 1985;9:31-34.

8. Etuk EU. Comparative study of the in vivo response of malaria parasites to chloroquine among the urban and rural dwellers in Sokoto State, Nigeria. Journal of Medical Sciences. 2005;5:159-162.

9. Olaleye BO, Williams LA, Allessandro UD, et al. Clinical predictors of malaria in Gambia children with fever or a history of fever. Trans R Soc Trop Med Hyg. 1998;92:300-304.

10. Sowunmi A, Akindele JA. Presumptive diagnosis of malaria in infants in an endemic area. Trans $R$ Soc Trop Med Hyg. 1993;87:422.

11. Waller D, Krishna S, Crawley J, et al. Clinical features and outcome of severe malaria in Gambian children. Clin Infect Dis. 1995;21:577587.

12. Miller LH, Baruch DI, Marsh K, Doumbo OK. The pathogenesis basis of malaria. Nature. 2002;415:673-679.

13. Woodrow CJ, Haynes RK, Krishna S. Artemisinins. Postgrad Med J. 2005;81:71-78. 